\title{
Transcriptomic responses to hypoxia in endometrial and decidual stromal cells
}

\author{
Kalle T Rytkönen ${ }^{1,2,3,4}$, Taija Heinosalo' ${ }^{1}$, Mehrad Mahmoudian ${ }^{2,5}$, Xinghong Ma ${ }^{3,4,6}$, \\ Antti Perheentupa ${ }^{1,7}$, Laura L Elo ${ }^{2}$, Matti Poutanen ${ }^{1}$ and Günter P Wagner ${ }^{3,4,8,9}$ \\ ${ }^{1}$ Institute of Biomedicine, Research Centre for Integrative Physiology and Pharmacology, University of Turku, Turku, \\ Finland, ${ }^{2}$ Turku Bioscience Centre, University of Turku and Abo Akademi University, Turku, Finland, ${ }^{3}$ Yale Systems \\ Biology Institute, West Haven, Connecticut, USA, ${ }^{4}$ Department of Ecology and Evolutionary Biology, Yale University, \\ New Haven, Connecticut, USA, ${ }^{5}$ Department of Future Technologies, University of Turku, Turku, Finland, ${ }^{6}$ College of \\ Life Sciences, Northeast Agricultural University, Harbin, China, ${ }^{7}$ Department of Obstetrics and Gynecology, Turku \\ University Hospital, Turku, Finland, ${ }^{8}$ Department of Obstetrics, Gynecology and Reproductive Sciences, Yale \\ Medical School, New Haven, Connecticut, USA and ${ }^{9}$ Department of Obstetrics and Gynecology, Wayne State \\ University, Detroit, Michigan, USA
}

Correspondence should be addressed to K T Rytkönen; Email: katury@utu.fi

\begin{abstract}
Human reproductive success depends on a properly decidualized uterine endometrium that allows implantation and the formation of the placenta. At the core of the decidualization process are endometrial stromal fibroblasts (ESF) that differentiate to decidual stromal cells (DSC). As variations in oxygen levels are functionally relevant in endometrium both upon menstruation and during placentation, we assessed the transcriptomic responses to hypoxia in ESF and DSC. In both cell types, hypoxia-upregulated genes in classical hypoxia pathways such as glycolysis and the epithelial mesenchymal transition. In DSC, hypoxia restored an ESF-like transcriptional state for a subset of transcription factors that are known targets of the progesterone receptor, suggesting that hypoxia partially interferes with progesterone signaling. In both cell types, hypoxia modified transcription of several inflammatory transcription factors that are known regulators of decidualization, including decreased transcription of STATs and increased transcription of CEBPs. We observed that hypoxia-upregulated genes in ESF and DSC had a significant overlap with genes previously detected to be upregulated in endometriotic stromal cells. Promoter analysis of the genes in this overlap suggested the hypoxiaupregulated Jun/Fos and CEBP transcription factors as potential drivers of endometriosis-associated transcription. Using immunohistochemistry, we observed increased expression of JUND and CEBPD in endometriosis lesions compared to healthy endometria. Overall, the findings suggest that hypoxic stress establishes distinct transcriptional states in ESF and DSC and that hypoxia influences the expression of genes that contribute to the core gene regulation of endometriotic stromal cells.

Reproduction (2020) 160 39-51
\end{abstract}

\section{Introduction}

Human reproductive success depends on a properly differentiated (decidualized) uterine endometrium that allows implantation, the formation of the placenta, and maintenance of the pregnancy (Gellersen \& Brosens 2014, Vinketova et al. 2016). In humans, and other catarrhine primates, decidualization of endometrial stromal fibroblasts (ESF) to endometrial stromal cells (DSC) takes place spontaneously during every menstrual cycle. Decidualization involves substantial transcriptional and cellular remodeling, enabling implantation and placental development as well as menstruation-associated renewal of endometrium (Gellersen \& Brosens 2014). This process is triggered by autocrine and paracrine signaling pathways dependent on progesterone activated progesterone receptor (PGR) and cyclic AMP (CAMP) mediated activation of protein kinase A (PKA) (Pavličev et al. 2017, Wu et al. 2018). These, together with expression of transcription factors (TFs) including forkhead box protein O1 (FOXO1), homeobox (HOX), and signal transducer and activator of transcription (STAT) paralogs contribute to the regulatory programming necessary for decidualization (Gellersen \& Brosens 2014, Vinketova et al. 2016).

The endometrium is exposed to hypoxic periods specifically upon menstruation as well as during placentation (Pringle et al. 2010, Maybin \& Critchley 2015), but the associated transcriptional regulation remains poorly characterized. A recent study assessed the role of hypoxia in menstrual repair (Maybin et al. 2018), but the difference in the hypoxia-related 
transcriptional regulation between undifferentiated ESF and differentiated DSC has not been studied. Moreover, it was recently shown that DSC-specific gene regulatory network involves factors that are part of the oxidative stress responses (Erkenbrack et al. 2018), placing a specific interest on oxygen-dependent gene regulation in the endometrium.

Importantly, endometrial responses to hypoxia are also relevant to several aspects of reproductive health. In endometriosis, endometrial cells grow outside of uterus in niches that are often more hypoxic than the highly vascularized uterus (Bishop 1956, Bourdel et al. 2007, Wu et al. 2019). Endometriosis lesions grow faster in hypoxia (Lu et al. 2014) and are associated with angiogenesis (Lu et al. 2014) and hormone actions, including regulation of estrogen receptor $(\mathrm{Wu}$ et al. 2012), are affected by hypoxia. Additionally, the abnormalities in pregnancy disorder preeclampsia are partly driven by hypoxia signaling (Tal 2012).

Up to date, no whole genome studies are available that describe the transcriptomic responses to hypoxia in the endometrial stromal cells. Here, we characterize the transcriptomic responses to severe hypoxia $(1 \%$ $\mathrm{O}_{2}, 24 \mathrm{~h}$ ) in cultured ESF and DSC. We assess the hypoxia-regulated pathways by enrichment analysis and specifically focus on hypoxia-regulated transcription factors. Further, we show that hypoxia-upregulated genes have significant overlap with genes known to be upregulated in endometriosis, and guided by promoter analysis of transcription factor binding sites, we select two transcription factors, JunD Proto-Oncogene (JUND) and CCAAT Enhancer Binding Protein Delta (CEBPD), for immunohistochemistry in endometriosis lesions and healthy endometria.

\section{Materials and methods \\ Cell culture and hypoxia treatment}

Human immortalized endometrial stromal fibroblasts (ESF) (T HESC, Mor lab, Yale University, corresponding to ATCC CRL-4003) were grown in normoxia $\left(21 \% \mathrm{O}_{2}\right)$ in Dulbecco's Modified Eagle's medium (DMEM) (Sigma-Aldrich, D2906), supplemented with $10 \%$ charcoal-stripped calf serum (Hyclone), 1\% antibiotic/antimycotic (ABAM; Gibco), 1nM sodium pyruvate (Gibco), $0.1 \%$ insulin-transferrin-selenium (ITS premix, BD Biosciences), and $0.12 \%$ sodium bicarbonate. To generate DSC, ESFs were decidualized by adding of $0.5 \mathrm{mM}$ 8-bromoadenosine 3',5'-cyclic monophosphate (8-Br-cAMP) (Sigma) and $0.5 \mu \mathrm{M}$ of the synthetic progestin medroxyprogesterone acetate (MPA) in DMEM supplemented with $2 \%$ charcoal-stripped calf-serum.

For ESF, hypoxia exposure was conducted for $24 \mathrm{~h}$ using ProOx C21 nitrogen-induced hypoxia system (BioSpherix, Red Field, $\mathrm{NY}$ ) at $1 \% \mathrm{O}_{2}, 5 \% \mathrm{CO}_{2}$ and compared to normoxic ESF from the same cell batch. For DSC, ESF were first decidualized for $40 \mathrm{~h}$ and then similarly exposed to hypoxia for $24 \mathrm{~h}$ and compared to normoxic DCS from the same cell batch that were decidualized for 2 days (Supplementary Fig. 1, see section on supplementary materials given at the end of this article). This protocol represents a reasonable approximation for the comparison, on one hand the DSC cells before hypoxia exposure were very close to the decidualization state of control normoxic DSCs and on the other hand this takes into account that process of decidulization also continues with lower pace during the hypoxia exposure, as hypoxia slows down cellular processes. Each of the four sample groups had two biological replicates.

\section{$R N A$-seq, differential transcription and visualization}

Total RNA was extracted with RNeasy Mini or Midi RNAextraction kits (QIAGEN) followed by on-column DNase I treatment. Total RNA quality was assayed with a Bioanalyzer 2100 (Agilent) and 500 ng of RNA samples were sequenced with Illumina Genome Analyzer II platform. For each sample, at least 30 million reads were acquired and quality parameters were checked with FastQC (http://www.bioinformatics.babr aham.ac.uk/projects/fastqc). Sequencing data are available in NCBI Gene Expression Omnibus (GEO; https://www.ncbi. nlm.nih.gov/geo/) under accession numbers GSE111570 (GSM3034449, GSM3034450, GSM3034451, and GSM3034452) and GSE63733 (GSM1556296, GSM1556297, GSM1556298, and GSM1556299). All data are also available from authors upon request.

Sequence reads were mapped to the GRCh37 human reference genome using Tophat2 (Trapnell et al. 2009) and the gene counts were calculated using HTSeq (Anders et al. 2015) according to Ensembl annotation (GRCh37.69) and normalized as transcripts per million (TPM) (Wagner et al. 2013). Differential transcription was analyzed using edgeR with upper quartile normalization (Robinson et al. et al. 2010) and the following cut-offs: false discovery rate (FDR) $<0.01$, absolute fold-change $(\mathrm{FC})>2.0$, and TPM $>2$. The principal component analysis (PCA) across the four conditions after edgeR upper quartile normalization indicated that the two biological replicates in each condition grouped tightly together (Supplementary Fig. 2).

For visualization, gene heatmaps were produced from averages of the absolute TPM values using pheatmap_1.0 in R 3.5. Hierarchical clustering of the genes was performed using Euclidean distance and the complete linkage method. TF set was constructed by combining genes in Ingenuity Pathway Analysis (IPA, Qiagen, www.qiagen.com/ingenuity) categories 'transcription regulator' and 'ligand-dependent nuclear receptor'. Hypoxia-regulated transcription factor subsets were intersected with PGR targets that were previously detected using siRNA by Demayo lab (we filtered the originally reported siPGR set using FC >2) (Mazur et al. 2015). A circos plot was produced in METASCAPE (metascape.org).

\section{Pathway analysis}

For gene ontology (GO) and gene set enrichment analysis and heatmaps, gene lists of differentially transcribed genes (FDR $<0.01$, FC $>2.0$, TPM $>2$ ) were used as input for METASCAPE, and available pathway databases (GO Biological Processes, 
Reactome Gene Sets, Canonical Pathways, Biocarta Gene Sets, KEGG Pathway, and Hallmark Gene Sets) were selected for analysis. We used 'IPA Canonical Pathways' tool to visualize enriched pathways.

\section{Endometriosis transcriptome data and statistical tests of the overlaps}

In order to test the significance of our cell culture results in a clinically relevant hypoxic niche, we investigated the overlap of hypoxia differentially transcribed genes with most relevant available endometriosis data from literature and databases. Endometriosis data sets included a stromal dataset (FACS isolated with CD10 antibody) of differentially expressed genes between endometriosis lesions vs control healthy endometrium (Rekker et al. 2017, Supplementary Table 1); a heterogeneous tissue dataset of differentially expressed genes in the endometrium of endometriosis patients vs healthy controls (Tamaresis et al. 2014, Supplementary Table 5 , endometriosis and abnormal) and endometriosis related genes listed in the DisGeNET v5.0- database (http://www. disgenet.org/) (Piñero et al. 2017). For the first two sets, lists of endometriosis-upregulated and endometriosis-downregulated genes were analyzed separately, whereas for the database one, list of all endometriosis-regulated genes was used. The statistical significance of the overlaps was determined using Fisher's exact test. The genes of the two most significant overlaps were further analyzed with pathway analysis and transcription factor binding site motif analysis.

\section{Transcription factor binding site (TFBS) analysis}

We tested the enrichment of TFBS in the promoters of the endometriosis-upregulated subsection of all the hypoxiaupregulated genes using GeneXplain 4.0 (http://genexplain. com). The set of all hypoxia-upregulated genes was used as the background and 'Search for enriched TFBSs (genes)' function was run with default settings (promoter: -1000 to +100 ). Two TFBS databases were searched: prediction based TRANSFAC_ public_vertebrates and ChIP-seq based GTRD_moderate. Both result lists were ranked with 'Site FDR' to inspect the most significantly enriched TFBS motifs. Top 20 enriched motifs were manually examined for corresponding TFs homologs among the top ESF and DSC hypoxia-upregulated TFs. As TFBS motif names and TF names do not constitute one-to-one correspondence, corresponding homologs (and synonyms) were manually defined using https://www.genecards.org/.

\section{Immunohistochemistry}

Patient samples were collected and processed as described previously (Heinosalo et al. 2018). Briefly, the Joint Ethics Committee of Turku University and Turku University Hospital approved collections and all study subjects provided written informed consent prior to recruitment. Endometriosis samples (stage IV deep infiltrating lesions: rectovaginal septum, sacrouterine ligament, and bladder) were collected during laparoscopy or laparotomy from three women (ages 31, 35, and 39), also eutopic endometrial biopsies were collected from the subjects. As a control group, endometrial biopsies from three healthy, endometriosis-free women (ages 37, 46, and 48) undergoing laparoscopic tubal ligation were collected. Histological evaluation was performed to confirm the presence of normal endometrial histology in controls and patients and to diagnose endometriosis in patients. All the samples studied were collected during the proliferative phase of the menstrual cycle from women that had not used any hormonal medication. Women with other significant disease or medication, suspicion of malignancy, pregnancy, or acute infection were excluded. Tissue samples were fixed in formalin and embedded in paraffin for histological analysis. Antigen retrieval of hydrated $5-\mu \mathrm{m}$ thick sections was performed in $10 \mathrm{mM}$ sodium citrate buffer ( $\mathrm{pH}$ 6.0), followed by immunohistochemistry with primary antibodies against JUND (mouse monoclonal, Jun D Antibody (D-9), sc-271938, Santa Cruz Biotechnology) with 1:1500 dilution and CEBPD (rabbit polyclonal Anti-CEBP Delta antibody ab65081, Abcam) with 1:250 dilution. Negative control samples not incubated with primary antibodies did not show any staining. Three samples in each sample group (endometriosis lesion, patient endometrium, and healthy control endometrium) were stained, and final lesion samples included rectovaginal septum and sacrouterine ligament from one patient (age 31) and bladder from another patient (age 39). Sections were scanned for analyses with the panoramic 250 Flash series digital slide scanner (3DHISTECH, Hungary). Control endometrial biopsies and eutopic endometrial biopsies from patients gave similar results, thus only representative IHCs from control endometrial biopsies are displayed.

\section{Results \\ Global transcriptomic effects of hypoxia}

We performed global RNA-seq from hypoxia treated $\left(1 \% \mathrm{O}_{2}, 24 \mathrm{~h}\right)$ immortalized human endometrial stromal fibroblasts ESF (ATCC CRL-4003) and 2-day decidualized (MPA, 8-br-cAMP) endometrial stromal cells (DSC) and compared these to corresponding normoxic conditions. Hypoxia-related upregulation of transcription (FDR $<0.01$, $\mathrm{FC}>2$ and TPM $>2$ in hypoxic condition) involved more shared genes between ESF and DSC compared to hypoxiarelated downregulation of transcription (FDR $<0.01$, FC $<-2$ and TPM $>2$ in normoxic condition) (Fig. 1A). Specifically, 36\% (728) of the genes upregulated were shared in both ESF and DSC, whereas 628 and 643 genes, respectively, were upregulated in a cell-type-specific manner. In contrast, $69 \%$ of the genes downregulated in DSC (1304) were DSC specific (901).

\section{Functional pathways affected by hypoxia}

Pathway enrichment analysis revealed a clustering pattern of functional terms that is concordant with the described gene proportions. In both cell types, the most significant cellular functions predicted to be upregulated by hypoxia ('hypoxia' and 'epithelial mesenchymal 
A
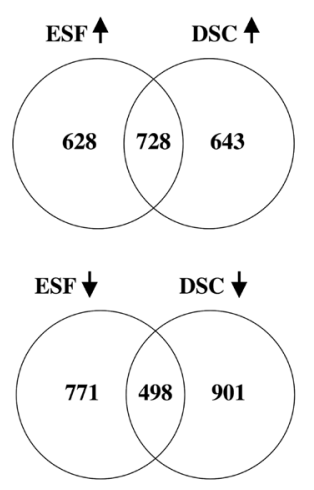

B

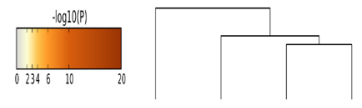

epithelial mesenchymal transition (H) hypoxia $(\mathrm{H})$

tissue morphogenesis (G)

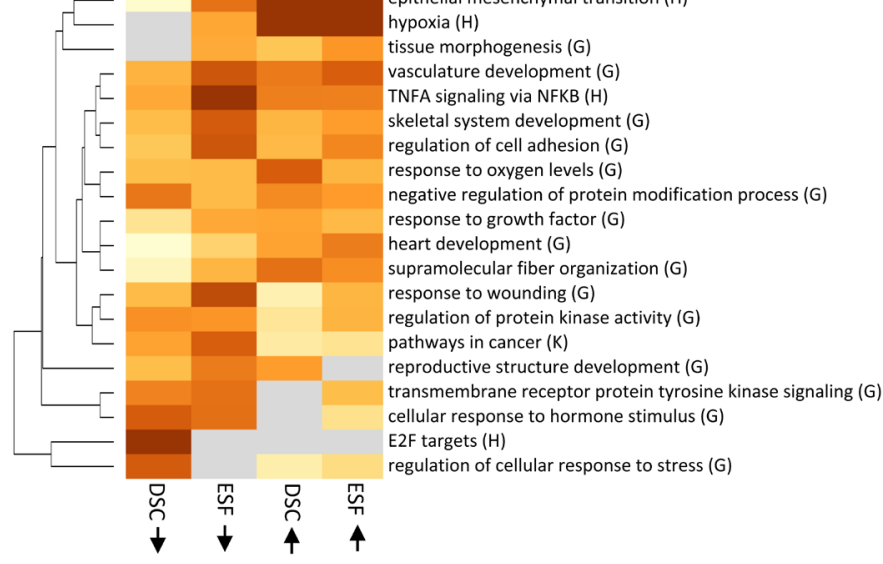

C

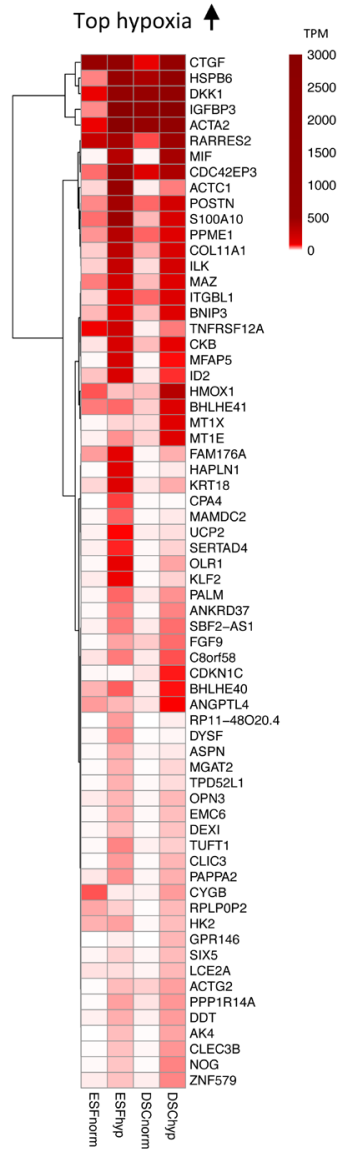

D Tор ЕMT $\uparrow$

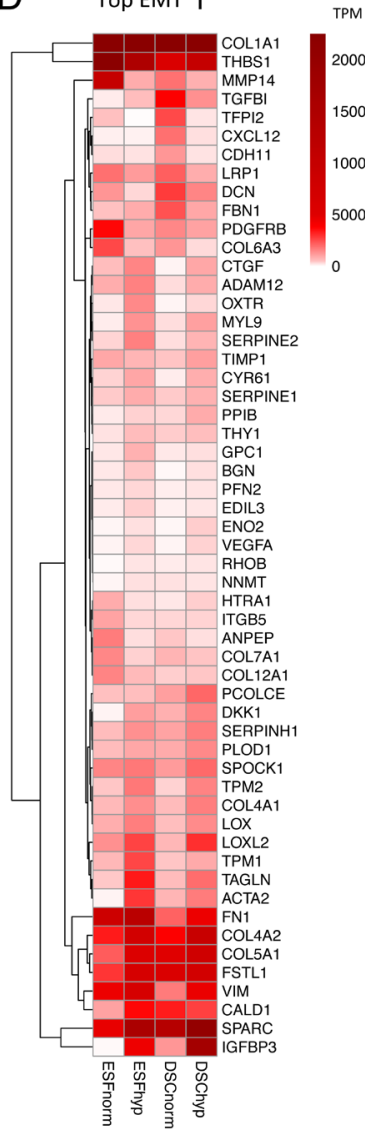

E

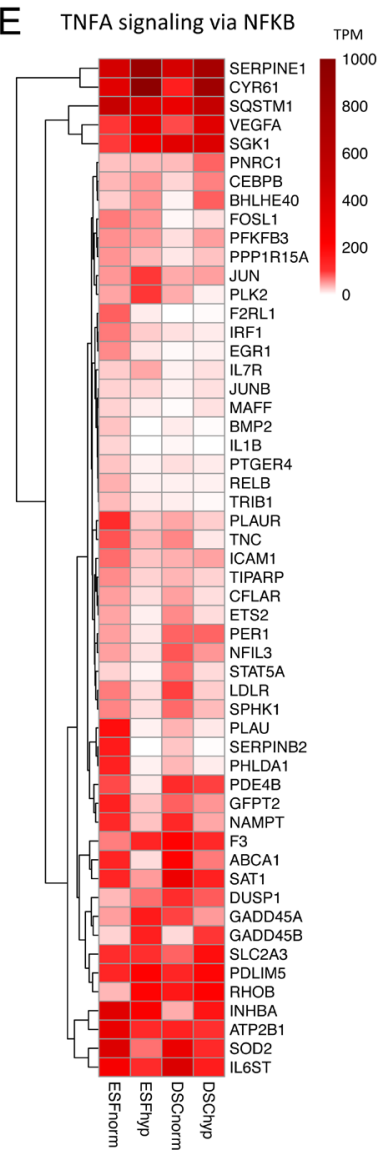

Figure 1 Hypoxia-regulated gene pathways in endometrial stromal fibroblasts (ESF) and endometrial stromal cells (DSC). (A) Differentially transcribed genes in hypoxia $\left(1 \% \mathrm{O}_{2}, 5 \% \mathrm{CO}_{2}, 24 \mathrm{~h}, \mathrm{FC}>2, \mathrm{FDR}<0.01, \mathrm{TPM}>2\right)$. Arrows up mark upregulated genes and arrows down mark downregulated genes. (B) Hierarchical clustering of the functional enrichment categories from METASCAPE ( $P$ values, default; see Supplementary Table 1) using the genes with significantly increased and decreased transcription as an input. The functional categories are from GO Biological Processes (G), Hallmark gene sets (H), and KEGG (K). Arrows mark the upregulated or downregulated genes in the four conditions. (C) Top 40 genes with increased transcription in hypoxic conditions in ESF and DSC (norm = normoxia, hyp=hypoxia), presented using hierarchical clustering (Euclidean, complete) of averages of absolute TPM values (>20 TPM). (D) 'Epithelial mesenchymal transition' (EMT) term associated hypoxia-upregulated and highly transcribed genes (>200 TPM). (E) All 'TNFA signaling via NFKB' term associated genes differentially transcribed under hypoxia (>20 TPM). TNFA, Tumor Necrosis Factor A; NFKB, Nuclear Factor Kappa B. See Supplementary Table 1 for the full list of hypoxia-regulated genes, the gene lists of detected enrichment categories, and TPM values used for the figures. 
transition') were clustered together (Fig. 1B). On the other hand, hypoxia-downregulated genes in ESF and DSC formed distinct enrichments, in ESF the most significant terms being 'TNFA signaling via NFKB' and 'response to wounding', whereas in DSC these were 'E2F targets' (involved in cell cycle regulation) and 'regulation of cellular response to stress' that were not detected in ESF.

As expected, in both cell types, hypoxiaupregulated genes included canonical glycolysis pathway (Supplementary Fig. 3) and other known target genes of hypoxia inducible factor 1 (HIF1alpha) (Fig. 1C). These genes, which also shared the highest hypoxic increases in both conditions, included insulin-like growth factor binding protein 3 (IGFBP3), macrophage migration inhibitory factor $(M I F)$, and metallothionein genes (MT1E, MT1X). MIF is a cytokine potentially regulating both inflammatory status and angiogenesis (Hahne et al. 2018), and MT1s are involved in the protective responses to oxidative stress (Xue et al. 2012). Upon hypoxia in DSC, of the classic decidualization markers, transcription of prolactin remained on the same level as in normoxia (TPM 7.9 to 5.4), whereas IGFBP1 was markedly downregulated (TPM 17 to 0.4 ) suggesting that hypoxia may interfere with metabolic aspects of decidualization. Most notably, in hypoxia, the TPM for IGFBP3, a paralog of IGFBP1, increased from 38 to 4382 TPM in ESF and from 1216 to 16865 TPM in DSC. IGFBPs bind to and modulate insulin-like growth factors (IGFs) and may affect glucose uptake and proliferation of the cells. Also, LDHA was highly transcribed and 8.6-fold upregulated in ESF (to TPM 1645) and 5.3-fold upregulated in DSC (to TPM 1381) (Supplementary Table 1A), confirming the extensive hypoxic metabolism and glycolysis.

In both cell types of our study, the top hypoxiaupregulated genes with high transcription levels included several extracellular matrix components and their regulators, including several collagens $(1 \mathrm{~A} 1,4 \mathrm{~A} 1,4 \mathrm{~A} 2$, $5 \mathrm{~A} 1$ ), fibronectin (FN1), vimentin (VIM), lysyl oxidases (LOX, LOXL2), and proteases that are involved in EMT (Fig. 1D) and that are relevant markers of mesenchymallike state in ESF (Yu et al. 2016, Owusu-Akyaw et al. 2019). Thus, knowing that these genes are central for both EMT and the opposite process, mesenchymal epithelial transition (MET), these results suggest that hypoxia may repress mesenchymal epithelial transition (MET) -like extracellular modifications typical for early decidualization.

In ESF, hypoxia repressed genes connected to inflammation, under the terms such as 'TNFA signaling via NFKB' and 'response to wounding' (Fig. 1B). This repression included the downregulation of proinflammatory cytokines, such as $I L 1 B$ and $B M P 2$, and TFs RELB, F2RL1, and IRF1 (Fig. 1E). These results suggest that hypoxia modifies the pro-inflammatory signaling characteristic for early decidualization (Salker et al. 2012, Rytkönen et al. 2019). In DSC, hypoxia-downregulated genes in the 'E2F targets' term include several regulators of cell cycle, chromosome segregation, and nuclear division (Supplementary Table 1B). The DSC-downregulated genes within the term 'regulation of cellular response to stress' include genes from the intracellular protein kinase signaling networks such as MAPK cascade (Supplementary Table 1B). For DSC, it has to be noted that the 2-day decidualization protocol likely reflects the decidualization state of early/mid secretory phase of the in vivo endometrium and that these phases are not likely as hypoxic as our hypoxia exposures (Maybin \& Critchley 2015, Maybin et al. 2018).

\section{Hypoxia-regulated transcription factors}

The TFs upregulated in response to hypoxia included several common mediators of hypoxia-induced gene repression. Of the TFs upregulated in ESF by hypoxia, $60 \%(66 / 110)$ were also upregulated in DSC, whereas of the downregulated TFs only $37 \%$ (37/100) were shared (Fig. 2A). In both cell types, hypoxia-upregulated transcriptional repressors, such as Inhibitor Of DNA Binding (ID) genes that repress the expression of other TFs (Fig. 2B).

The master hypoxic regulators (HIF-1alpha and EPAS1, a.k.a. HIF-2alpha) were highly transcribed in all conditions, but were downregulated in DSC by hypoxia 5- and 3-fold, respectively (Fig. 2C). In DSCs, the hypoxia responses were also linked to the regulation of circadian rhythm, notably, the transcriptional repressors BHLHE4O (DEC1) and BHLHE41 (DEC2) were highly upregulated by hypoxia, 17 - and 11 -fold, respectively (Fig. 2B). These factors repress TFs regulating circadian rhythm, such as PER and CLOCK. Consistent with this result, we observed downregulation of $P E R 1 / 3$ in ESF and CLOCK in DSC under the same conditions (Supplementary Table 1A).

The effects of hypoxia on core regulators of decidualization were multifaceted. In both cell types, but particularly in DSC, HOXA10, HOXA11, CEBPB, and $C E B P D$ were upregulated by hypoxia (Fig. $2 \mathrm{~B}$ and Supplementary Table 1A), whereas FOXO1, STAT3, and STAT5A were downregulated (Fig. 2C). Of these, especially CEBPs and STATs are involved in the initial pro-inflammatory decidualization phase (Wang et al. 2012, Rytkönen et al. 2019).

Other notable upregulated groups of TFs included members of Jun/Fos family (JUND and JDP2) and several Kruppel-like factors such as KLF2, KLF4, and KLF7, of which KLF2 was 18-fold upregulated in ESF. KFL2 is a known negative regulator of NFKB pathway (Jha \& Das 2017) and potentially participates in the of the observed ESF-specific NFKB pathway repression under hypoxia. 


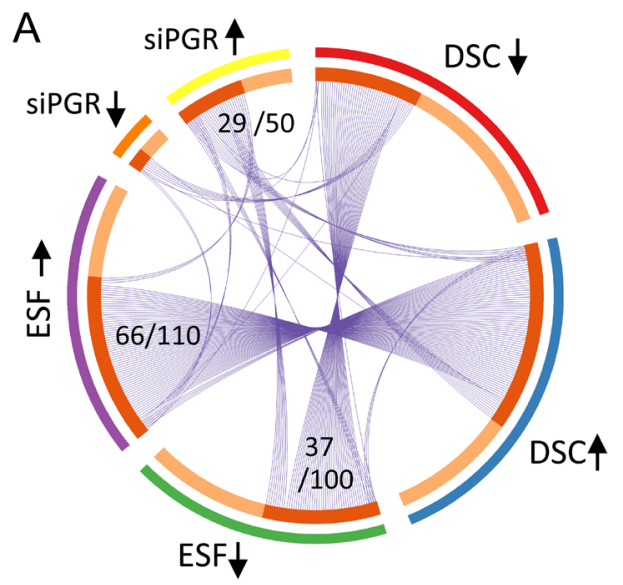

B
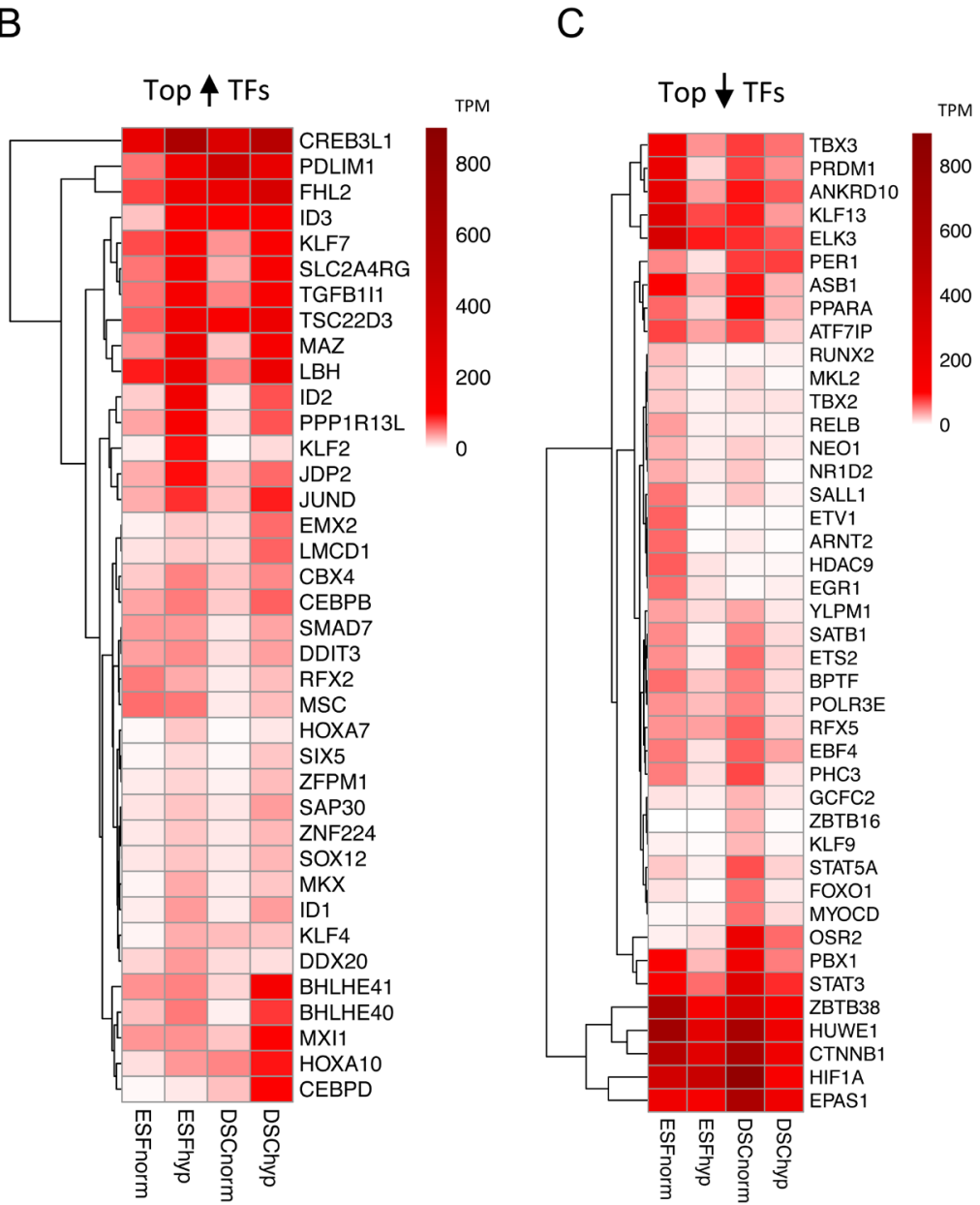

Figure 2 Top hypoxia-regulated transcription factors (TF) in endometrial stromal fibroblasts (ESF) and endometrial stromal cells (DSC). (A) Differentially transcribed TFs in hypoxia $\left(1 \% \mathrm{O}_{2}, 5 \% \mathrm{CO}_{2}, 24 \mathrm{~h}, \mathrm{FC}>2, \mathrm{FDR}<0.01, \mathrm{TPM}>2\right)$ and TFs regulated by decidualization regulator progesterone receptor (PGR) PGR siRNA (Mazur et al. 2015) (FC >2). Arrows up mark upregulated genes and arrows down mark downregulated genes. Top 25 TFs with increased (B) or decreased (C) transcription in ESF and DSC under hypoxia (norm=normoxia, hyp=hypoxia) presented using hierarchical clustering (Euclidean, complete) of averages of absolute TPM values (>20 TPM). (D) 29 Hypoxia-regulated (ESF and/or DSC) and PGR-downregulated (siRNA upregulated) TFs presented using hierarchical clustering (Euclidean, complete) of averages of absolute TPM values (>20 TPM). *Notes a subset of highly transcribed and PGR downregulated TFs for which hypoxia partly restores the ESF-like

transcriptional state (FOSL1, KLF6, BHLHE41, HMGA1, ETV5). FOSL1, FOS Like 1, AP-1 Transcription Factor Subunit; KLF6, Kruppel Like Factor 6; BHLHE41, Basic Helix-Loop-Helix Family Member E41 (DEC2); HMGA1, High Mobility Group AT-Hook 1; ETV5, E26 transformation-specific Variant 5. See Supplementary Table 2 for the list of hypoxia- and PGR-regulated TFs and the lists of the specific intersections with corresponding TPM values.

\section{Hypoxia interferes with progesterone-receptor- regulated TF networks}

In both cell types, hypoxia downregulated several genes that were under the GO term 'cellular response to hormone stimulus' (Fig. 1B). Signaling via progesterone receptor (PGR) is known to be a main driver of decidualization, and it regulates, for example, the expression of FOXO1, HOXO10, CEBPs, and STATs. We, thus, investigated the effect of hypoxia specifically in PGR-regulated TFs by intersecting ChIP-seq detected and PGR-regulated ( $>2$-fold) TFs from a previous study (Mazur et al. 2015) with the hypoxia-regulated TFs. The data revealed that $8 / 18$
PGR-upregulated and 29/50 PGR-downregulated TFs were influenced by hypoxia (Fig. 2A). This suggests that primarily TF networks downregulated by PGR are modified by hypoxia. Notably, of the 29 TFs downregulated by PGR, 13 showed similar downregulation by hypoxia in ESF, while 15 were upregulated in DSC by hypoxia (Fig. 2A and D). The data also suggest that, in DSC, hypoxia partly restores the ESF-like transcriptional state for a subset of highly transcribed and PGR-downregulated TFs (Fig. 2D). Overall, these observations suggest that hypoxia, at least partly, reverses PGR dependent transcriptional changes during early decidualization. 


\section{Relationship between gene sets upregulated by hypoxia and in endometriosis}

In order to explore the significance of our cell culture results for a clinically relevant hypoxic niche, we investigated the overlap between the genes differentially transcribed in hypoxia with the data available on endometriosis using Fisher's exact test (Fig. 3A). The overlap with the highest significance was observed between the ESF hypoxiaupregulated genes and the endometriosis-upregulated genes $\left(P=5.8 \times 10^{-27}, 3.90\right.$-fold) from a dataset comparing isolated endometrial stromal cells from endometriosis lesions to healthy control endometrium (Rekker et al. 2017). In this dataset, also the overlap with DSC hypoxiaupregulated genes with endometriosis-upregulated genes was highly significant $\left(P=6.0 \times 10^{-19}, 3.25\right.$-fold). This extensive overlap of hypoxia-upregulated (ESF and DSC) genes of the endometriosis-upregulated genes 39\% (157/402, TPM >2 in our cell culture) suggests that gene regulatory programs responding to hypoxic conditions contribute to the gene regulation in the stroma of endometriosis lesions.

The overlaps with endometriosis tissue datasets that were not stromal-cell specific were not significant or less overlapping compared to the stromal-cell-specific data (Fig. 3A). For a heterogeneous tissue, dataset of differentially expressed genes in the endometrium of endometriosis patients vs healthy controls (Tamaresis et al. 2014), the overlaps of hypoxia- and endometriosis-upregulated genes were significant $\left(P=2.5 \times 10^{-11}-2.2 \times 10^{-5}, \quad 2.66\right.$-fold -1.92 -fold $)$. Further, the endometriosis related genes in DisGeNET v5.0 database (without the direction of regulation) had significant overlaps $\left(P=2.2 \times 10^{-13}-2.0 \times 10^{-4}, 2.58\right.$-fold -1.64 -fold) in both hypoxia-upregulated and hypoxiadownregulated genes.

We then used the 157 stromal-cell-specific genes that were hypoxia- and endometriosis upregulated as an input for pathways analysis (Fig. 3B). The most enriched categories included 'angiogenesis', 'epithelial mesenchymal transition', and 'estrogen response early' (Fig. 3C). The hypoxia and endometriosis-regulated 'angiogenesis' genes included several highly transcribed and secreted ECM and adhesion molecules such as CCN2 (Cellular Communication Network Factor 2), Serpine1, MCAM (Melanoma Cell Adhesion Molecule), and JCAD (Junctional Cadherin 5 Associated) (Fig. 3D). Additionally, the known core endometriosis TF GATA6 was hypoxia upregulated and the previously mentioned KLF2 (Kruppel Like Factor 2) was substantially upregulated in hypoxic ESF (Fig. 3D). Of the hypoxia and estrogen regulated genes, KRT8 and KRT18 are keratins
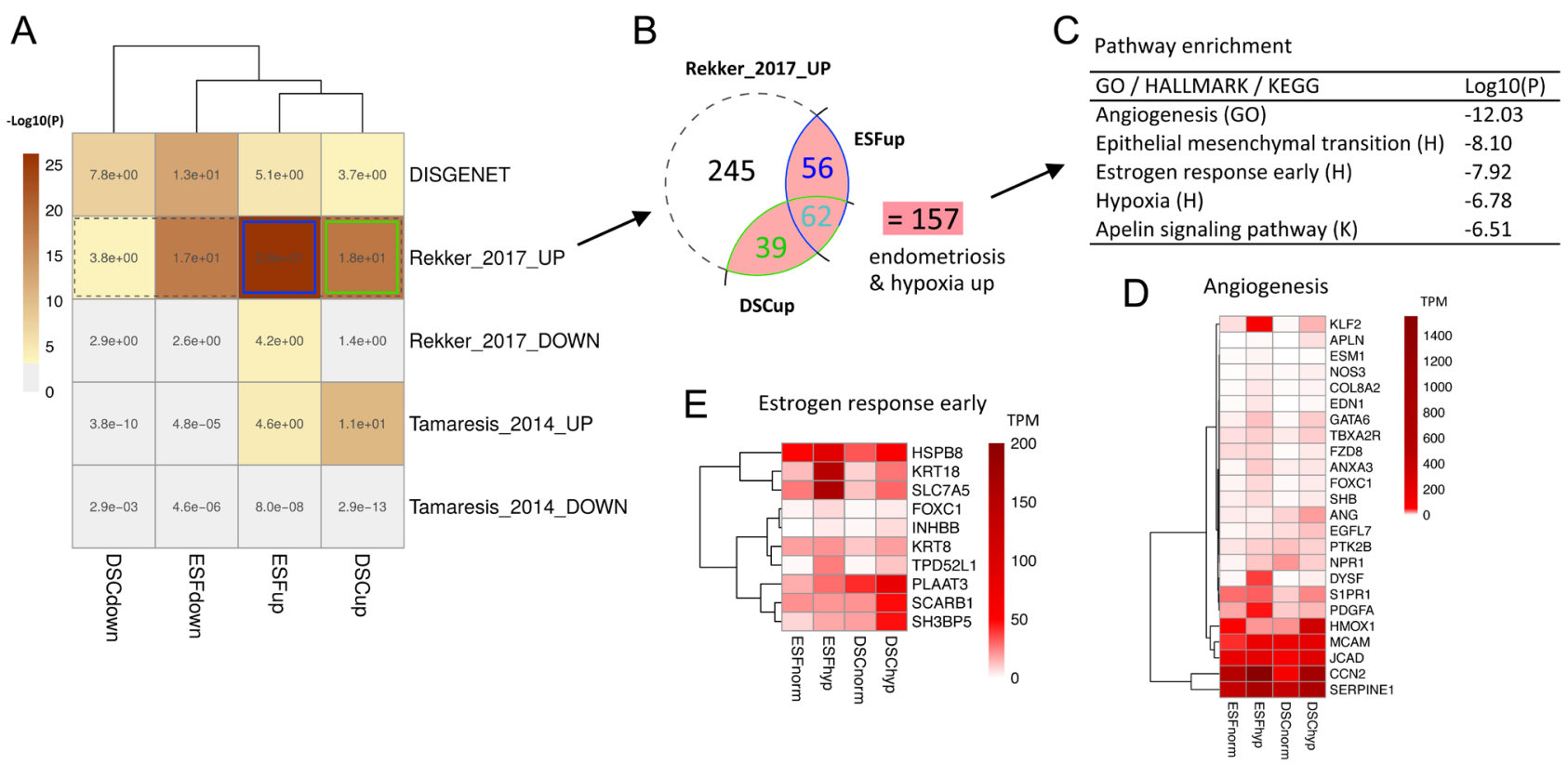

Figure 3 Overlaps of hypoxia-regulated and endometriosis-regulated genes. (A) Significance of the overlaps of hypoxia-regulated genes and three representative endometriosis datasets determined using Fishers exact test. Overlaps between hypoxia-upregulated genes with stromaspecific endometriosis-upregulated genes (Rekker et al. 2017) were highly significant (blue and green boxes). Other analyzed datasets were a whole tissue (cell type heterogeneous) endometrium dataset of endometriosis patients vs healthy controls (Tamaresis et al. 2014) and endometriosis associated genes from DisGeNET v5.0 database. (B) Venn diagram showing the number of genes in Rekker et al. (2017) endometriosis-upregulated genes (dashed line) with overlapping ESF and DSC hypoxia-upregulated genes (unique green and blue, respectively). Total of 157 genes that were hypoxia- and endometriosis regulated were then used as an input for pathway enrichment analysis (C), of which the top enrichment categories are displayed. (D) Heatmap of genes in the 'Angiogenesis' enrichment term. (E) Heatmap of genes in the 'Estrogen response early' enrichment term. The functional categories are from GO Biological Processes (GO), Hallmark gene sets (H), and KEGG (K). See Supplementary Table 3 for the original and overlapping gene lists, enrichment gene lists, and corresponding TPM values used for the heatmaps. 
that dimerize with each other to form intermediate filaments and SCL7A5 (Solute Carrier Family 7 Member 5 , or LAT1) is an amino-acid transporter (Fig. 3E).

\section{Promoter transcription factor binding site (TFBS) analysis of hypoxia- and endometriosis-upregulated genes reveals enrichment for Jun/Fos and CEBP motifs}

We next analyzed the promoters of these 157 hypoxiaand endometriosis-upregulated genes (Fig. 3B) to find TFs that potentially drive the hypoxic response in endometriosis. Using GeneXplain 4.0, we searched two databases, prediction based TRANSFAC and ChIP-seq based GTRD, for TFBS motifs enriched in the promoters $(-1000$ to +100 of TSS) of these 157 genes (Table 1$)$. Then, we manually examined the top 20 ranked (site FDR) motifs for corresponding TFs homologs among the highly hypoxia-expressed and upregulated TFs (from Fig. 2B). We discovered that there were motifs for the hypoxia-upregulated Jun/Fos family members JDP2 and JUND and CEBP family members CEBPB and CEBPD. Of these, JUND and CEBPD were selected for protein level validation in endometriosis biopsies due to their higher hypoxic upregulation compared to JDP2 and CEBPB (Fig. 2B) in ESF (JUND 3.2-fold, CEBPD 5.0fold) and in DSC (JUND 4.8-fold, CEBPD 5.2-fold) (see subsequent section).

The top motifs in the TFBS analysis that did not have corresponding hypoxia-upregulated TF homolog in our experiment included motifs for MEF2 (Myocyte Enhancer Factor 2), B-ATF (Basic Leucine Zipper ATF-Like TF), OCT1 (POU2F1, POU Class 2 Homeobox 1), and TBP/ TATA (TATA binding protein) (Table 1). Of these, MEF2C was 2.6-fold downregulated in ESF, whereas the others were not hypoxia regulated.

\section{Elevated immunohistochemical staining of JUND and CEBPD in deep endometriosis compared to endometrial biopsies}

Immunohistochemical staining of JUND and CEBPD was conducted in deep infiltrating endometriosis lesions and compared to endometrial biopsies (patients and healthy controls) (Fig. 4). The stromal staining of JUND was considerably stronger in endometriosis compared to control endometrium; however, in both, roughly only half of the cells were stained. Strong staining for JUND was present in all epithelial cells of the lesion, whereas in control endometrium, epithelial cell staining was weak. For CEBPD, we detected staining in most of the stromal and epithelial cells, and both cells had stronger staining in the endometriosis lesions compared to control endometria.

\section{Discussion}

It is known that the endometrium is exposed to hypoxic conditions specifically during menstruation as well
Table 1 Transcription factor binding site (TFBS) enrichment analysis in the promoters of hypoxia- (ESF and DSC) and endometriosisupregulated genes.

\begin{tabular}{|c|c|c|}
\hline & Site FDR & Hypoxia up \\
\hline \multicolumn{3}{|l|}{ TRANSCFAC } \\
\hline OCT1_04 & 4,1E-49 & na \\
\hline TATA_01 & 4,1E-49 & na \\
\hline MEF2_03 & $3,1 \mathrm{E}-38$ & na \\
\hline RSRFC4_01 & 1,7E-32 & na \\
\hline FOXJ2_02 & 1,5E-28 & na \\
\hline MEF2_01 & 1,7E-26 & na \\
\hline PBX1_01 & $8,4 \mathrm{E}-26$ & na \\
\hline MEF2_02 & $2,8 \mathrm{E}-25$ & na \\
\hline GATA2__01 & $2,4 \mathrm{E}-24$ & na \\
\hline HNF1_01 & $5,6 \mathrm{E}-23$ & na \\
\hline CDP_01 & $3,3 \mathrm{E}-21$ & na \\
\hline AP1_01 & 1,5E-20 & JDP2, JUND \\
\hline BRN2_01 & 1,3E-19 & na \\
\hline CEBP_Q2 & $2,0 \mathrm{E}-19$ & CEBPB, CEBPD \\
\hline AREB6_04 & 7,0E-19 & na \\
\hline EVI1_05 & $1,4 \mathrm{E}-17$ & na \\
\hline PAX4__04 & $1,6 \mathrm{E}-17$ & na \\
\hline OCT1_01 & $4,0 \mathrm{E}-17$ & na \\
\hline EN1_01 & $6,3 \mathrm{E}-17$ & na \\
\hline MEF2_04 & $2,0 \mathrm{E}-16$ & na \\
\hline \multicolumn{3}{|l|}{ GTRD } \\
\hline MEF-2A.1 & $7,5 \mathrm{E}-17$ & na \\
\hline B-ATF.1 & $7,5 \mathrm{E}-17$ & na \\
\hline MEF-2C.1 & $3,5 \mathrm{E}-15$ & na \\
\hline CEBPalpha.1 & $7,5 \mathrm{E}-13$ & CEBPB, CEBPD \\
\hline TBP.1 & 5,7E-10 & na \\
\hline STAT6.1 & 1,1E-06 & STAT4 \\
\hline SOX-2.1 & 1,1E-06 & SOX12 \\
\hline GATA-2.1 & 1,1E-06 & na \\
\hline C-Jun.1 & 2,9E-06 & JDP2, JUND \\
\hline TCF-7L1.1 & 6,7E-06 & na \\
\hline SMAD1.1 & $9,6 \mathrm{E}-06$ & SMAD7 \\
\hline CEBPdelta.1 & $1,1 \mathrm{E}-05$ & CEBPB, CEBPD \\
\hline FOXP2.1 & 1,4E-05 & FOXP4 \\
\hline PBX-1.1 & 1,6E-05 & na \\
\hline IRF-4.1 & 2,1E-05 & na \\
\hline PU1.1 & 2,3E-05 & na \\
\hline STAT5A. 1 & 7,9E-05 & STAT4 \\
\hline STAT1.1 & $2,8 \mathrm{E}-04$ & STAT4 \\
\hline GATA-3.1 & $3,0 \mathrm{E}-04$ & na \\
\hline PPARgamma & $3,2 \mathrm{E}-04$ & na \\
\hline
\end{tabular}

Both prediction based (TRANSFAC) and ChIP-seq based (GTRD) databases were searched for TFBS enrichment using the 157 hypoxia- and endometriosis-upregulated genes as foreground and all hypoxia-upregulated genes as a background. Top enriched TFBS motifs are displayed by 'Site FDR' rank and 'Hypoxia up' column displays the presence of a TF homologs corresponding to the TFBS motifs among top hypoxia-upregulated TFs (from Fig. 2B).

Endometriosis-upregulated genes are from Rekker et al. (2017)). See Supplementary Table 4 for the input gene lists and full TFBS enrichment lists.

as during placentation (Pringle et al. 2010, Maybin \& Critchley 2015). Here we conducted a global analysis of the transcriptomic responses to severe hypoxia in cultured immortalized ESF and DSC by comparing the hypoxia exposed $\left(1 \% \mathrm{O}_{2}, 24 \mathrm{~h}\right)$ cultures to normoxic controls. Overall, our findings suggest that hypoxia establishes related but distinct transcriptional states in ESF and 

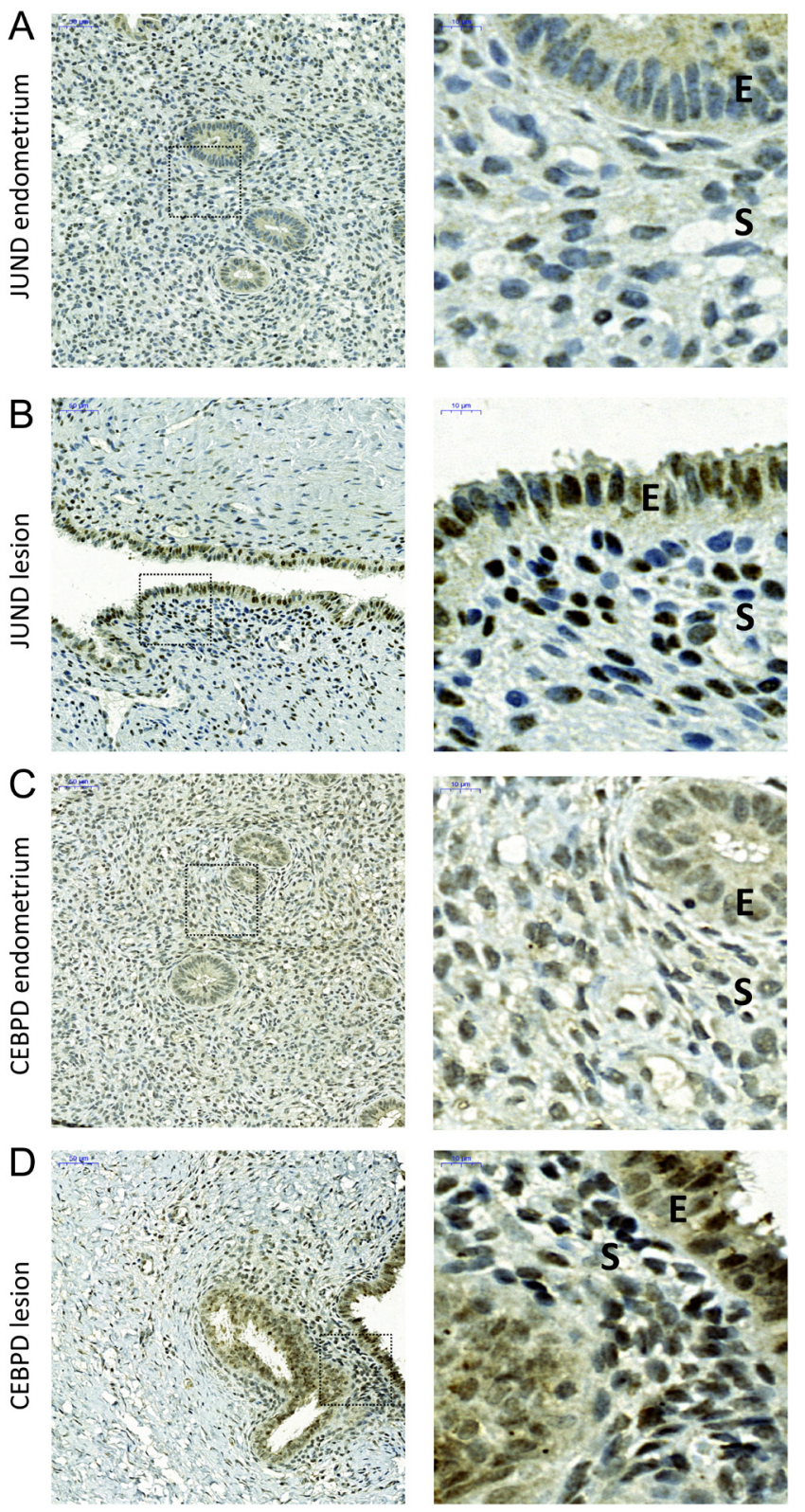

Figure 4 Immunohistochemical staining of JunD Proto-Oncogene (JUND) and CCAAT Enhancer Binding Protein Delta (CEBPD) in deep infiltrating endometriosis lesions and healthy control endometria. (A and B) Representative JUND staining shows increased expression in endometriosis lesions than control endometrium in both stromal cells and epithelial cells. (C and D) Representative CEBPD staining shows that CEBPD is expressed in both stroma and epithelium and is highly expressed in the stroma of endometriosis lesion. $\mathrm{E}=$ epithelium; $\mathrm{S}=$ stroma.

DSC. Several upregulated functional pathways, such as glycolysis and EMT, were shared with the two cell types, but many hypoxic responses, such as downregulation NFKB pathway in ESF and downregulation of stress responses as well as core hypoxic response genes in DSC, were cell-type specific. Further, specifically, as immortalized and not primary cell lines we used in the study, we inspected the translational aspects of our experiment using two strategies. First, we intersected the results of our hypoxia experiment with previous studies on endometriosis, endometriosis presenting a clinically relevant condition where stromal cells often inhabit hypoxic niches (Bishop 1956, Bourdel et al. 2007, Wu et al. 2019), and secondly, we investigated hypoxiaregulated transcription factors in the endometriosis and endometrium using immunohistochemistry.

Our results show that hypoxia interferes or modifies several gene regulatory networks necessary for early decidualization. In both ESF and DSC, hypoxia promotes fibroblastic phenotype (EMT) and, thus, may interfere with the development of the quasi-epithelial state of DSC. Further, when looking at TFs, the intersection of our results with PGR knockdown data (Mazur et al. 2015) suggests that hypoxia partly reverses PGR-dependent gene expression typical for early decidualization.

In both ESF and DSC, hypoxia enhanced classical hypoxia pathways including glycolysis and insulin-like growth factor (IGF) mediated signaling by altering the expression of IGF binding proteins (IGFBP1, IGFBP3, etc.). These modulate action of IGFs and thereby may affect glucose uptake and proliferation of the cells (Ding \& Wu 2018). Substantial hypoxic induction of IGFBP3 has also been observed in other cell types (Natsuizaka et al. 2012, Chang et al. 2015) and has been associated with robust hypoxic polysome enrichment of IGFBP3 mRNA that may permit continuous translation under hypoxia (Natsuizaka et al. 2012). In endometrial stromal cells, increased glycolytic metabolism appears to take place also in well oxygenated conditions (Kommagani et al. 2013), and the cells actively secrete lactate (Zuo et al. 2015). These observations can be also viewed in the context of Warburg effect or 'pseudohypoxia' (Russell et al. 2017), that is, that stromal cells robustly express hypoxia-associated proteins, such as glycolytic enzymes, regardless of oxygen levels. Moreover, recent studies on first-trimester decidua show that the uterine natural killer cell (uNK) populations that are critical for placental development also drive glycolysis (Vento-Tormo et al. 2018), suggesting that hypoxia (or pseudohypoxia) related metabolic programming including glycolysis is not limited to stromal cells and tumors but may be characteristic also to other cell types of decidualizing endometrium.

In our hypoxia experiment, expression of stress response genes appears to be reduced specifically in DSC, evidenced by general downregulation of stress response genes and the downregulation of mRNA levels of HIF-1alpha and HIF-2alpha, putatively representing negative feedback regulation via repressors such as upregulated ID genes (Inhibitor Of DNA Binding). Hypoxic downregulation of HIF mRNA in DSC can be viewed as part of general desensitized stress responses in DSC, as previously reported on cells exposed to oxidative stress (Kajihara et al. 2013) and other stress 
pathways (Muter et al. 2018) and may even be related to the previously mentioned Warburg effect in DSC, as glycolysis short cuts the mitochondria, which are major players in apoptosis. Transcriptional repression of HIFS do not contradict with the previously reported protein levels showing HIF2 stabilization during early secretory phase (Maybin et al. 2018), because HIFs are mainly regulated by hypoxia on post-translational level (Bishop \& Ratcliffe 2014). Additionally, in our study, of the several upregulated repressor TFs, DECs (BHLHE40 and BHLHE41) specifically repress CLOCK and PER genes (Sato et al. 2016), which were also downregulated in our experiment. These circadian rhythm TFs are involved in synchronizing decidualization-associated proliferation (Muter et al. 2015), and our data suggest that also this switch is modified by hypoxia.

As early decidualization is partly an inflammatory process, several of the early decidualization factors are also regulators of inflammation, including STAT and CEBP pathways. In both cell types, but especially in DSC, hypoxia downregulated FOXO1, STAT3, and STAT5A that are known initiators of decidualization (Gellersen \& Brosens 2014), thus suggesting that hypoxia suppresses the initial pro-inflammatory phase of decidualization. Hypoxia also upregulated AP1 factors (JUND and JDP2) that often mediate inflammation, but these are also general activators for fibroblast proliferation (Manabe et al. 2002, Florin et al. 2004). On the other hand, transcription factors that are known to be necessary for decidualization, such as HOXA10, HOXA11, CEBPB, and $C E B P D$ (Gellersen \& Brosens 2014), were upregulated by hypoxia, suggesting that hypoxic periods may also have positive effects for decidualization together with the potential to prime stromal cells for stress tolerance (Kajihara et al. 2013) or glycolytic metabolism that is enhancing decidualization (Kommagani et al. 2013, Zuo et al. 2015).

Hypoxic modification of decidualization related inflammatory states or progesterone signaling may also be relevant in reproductive disorders including endometriosis, where hypoxia has been show to induce stromal cell migration and enlargement of lesions (Lu et al. 2014, Liu et al. 2017). We investigated the relevance of our cell-culture results for endometriotic lesions by overlapping those with transcriptomic data from endometriosis and discovered a significant overlap of hypoxia-upregulated genes with endometriosisupregulated genes. The overlap was most pronounced in a dataset of isolated endometrial stromal cells (Rekker et al. 2017) in which endometriosis lesions were compared to healthy control endometrium. Notably, the high proportion of hypoxia-upregulated genes $(39 \%, 157 / 402)$ of the intersected endometriosisupregulated genes (Rekker et al. 2017) suggests that hypoxic gene regulatory programs extensively contribute to the gene expression in the stroma of endometriotic lesions.
The overlap of 157 upregulated genes in hypoxia and endometriotic stromal cell was enriched for genes promoting angiogenesis, EMT, and estrogen responses. Angiogenesis and estrogen response were not among the most enriched pathways in the primary analysis of hypoxia-upregulated genes (Fig. 1B) suggesting for the presence of endometriosis-specific hypoxic interactions that are notpresent in stromal cells originating from healthy endometrium. Our results support previous observations (Wu et al. 2012, 2019) that, in endometriosis, hypoxia and estrogen may synergistically promote the growth of the lesions. For example, the major angiogenic factor VEGFA was upregulated in our hypoxia experiment and is known to be regulated by both estrogen and hypoxia (Zhang et al. 2017). Among the angiogenesis enriched genes, we observed substantial hypoxic upregulation of KLF2, a known repressor of T-cells/monocyte activation and NFKB pathway (Jha \& Das 2017). Activation of NFKB pathway has been associated with the initiation of menstruation (Evans \& Salamonsen 2014), and in the context of endometriotic niche, the downregulation of NFKB pathway may contribute to the ability of stromal cells to escape the clearance by immune system.

In order to systemically search for hypoxiaupregulated TFs that drive endometriosis, we conducted promoter analysis of the overlap of 157 hypoxia- and endometriosis-upregulated genes. We discovered that Jun/Fos family members JDP2 and JUND and CEBP family members CEBPB and CEBPD were both robustly hypoxia upregulated and enriched in the promoters of the overlapping genes. Of these, we selected JUND and CEBPD for protein level studies in deep endometriosis and healthy control endometrium and found elevated immunohistochemical staining of JUND and CEBPD in deep endometriosis compared to controls.

Our study highlights JunD as a potential hypoxiaregulated intervention target in endometriosis. The upregulation of Jun/Fos pathway components in endometriosis has been reported in several studies (Hastings et al. 2006, Tamaresis et al. 2014), and previously, C-JUN NH2-terminal kinase inhibitor was reported to reduce endometriosis in baboons (Hussein et al. 2016). While cytokine profiling of endometriotic peritoneal aspirates, the detected Jun/Fos driven cytokine expression was previously interpreted as a major component of macrophage directed cytokine signature (Beste et al. 2014), our results suggest that endometriotic stromal cells that express Jun/Fos factors contribute to the cytokine signatures of endometriosis.

$C E B P D$, which in our study was five-fold induced by hypoxia in both ESF and DSC, has been suggested to act as a tumor suppressor that hypoxia turns into a growth promoter (Balamurugan \& Sterneck 2013). Our immunohistochemical results showed increased CEBPD protein levels in deep endometriosis lesions. This differs from a previous immunohistochemical assessment of CEBPD in extra-ovarian endometriosis (Yang et al. 2002), 
where no differential staining was reported between endometriosis and controls, potentially due to different types of lesions investigated. Further, CEBPD is a paralog of CEBPB, which is a known core regulator of decidualization (Wang et al. 2012, Tamura et al. 2017); however, both CEBPB and CEBPD target decidual PRL promoter (Pohnke et al. 1999). Recent transcriptomic analysis have indicated that CEBPD is early upregulated and late downregulated during decidualization, whereas CEBPB levels do not change after the initial upregulation (Rytkönen et al. 2019). Putatively, our observation of stronger hypoxia-dependent transcriptomic upregulation of CEBPD together with the promoter analysis suggests that CEBPD may have more pronounced regulatory role in endometriosis compared to CEBPB.

Recently described HIF binding regions provide a mechanistic perspective on hypoxia-induced changes in the Jun/Fos or CEBP expression (Smythies et al. 2019). For example, in HepG2 cells both HIF1 and HIF2 binding regions are enriched with CEBPD motifs (Smythies et al. 2019), and in multiple cell types, especially HIF2, binding regions are enriched with Jun/Fos motifs (Smythies et al. 2019). Indirectly, this suggests that, also in endometrial stromal cells, HIF2 is a potential interaction partner for Jun/Fos TFs. On the other hand, hypoxia-related regulatory interactions are not necessarily HIF mediated, but may proceed through changes in chromatin structure via oxygendependent histone lysine demethylases (KDM). For example, ER receptor is regulated by KDM3A (Wade et al. 2015), which is an oxygen-dependent histone demethylase. Recently, several KDMs, such as KDM5s (Batie et al. 2019) and KDM6s (Chakraborty et al. 2019), were reported to direct extensive hypoxiadependent transcriptional changes. Specifically, KDM6 activation has been associated with uterine fibroblast activation (Nancy et al. 2018), which is concordant with our observation that hypoxia promotes fibroblastic phenotype in endometrial stromal cells.

In conclusion, the transcriptome analysis performed in this study suggests that hypoxia enhances glycolytic energy metabolism and promotes fibroblastic phenotype (EMT) and, thus, may interfere with the development of the quasi-epithelial state of DSC. Hypoxia also modifies inflammatory pathways and partly counteracts PGR actions, suggesting that hypoxia affects regulatory networks that are essential for decidualization. Hypoxia-upregulated genes have significant overlap with previously detected endometriosis-upregulated stromal genes (Rekker et al. 2017), and promoter analysis of this overlap revealed that Jun/Fos and CEBP transcription factors are potential hypoxic drivers of transcription in endometriosis. Of these, we validated the increased expression of JUND and CEBPD in endometriosis lesions using immunohistochemistry. Overall, the findings suggest that hypoxic stress constitutes related but distinct transcriptional states in ESF and DSC and that hypoxic regulatory programs contribute to the core gene regulation in the stroma of endometriotic lesions.

\section{Supplementary materials}

This is linked to the online version of the paper at https://doi. org/10.1530/REP-19-0615.

\section{Declaration of interest}

The authors declare that there is no conflict of interest that could be perceived as prejudicing the impartiality of the research reported.

\section{Funding}

Research reported in this publication was supported by European Commission Horizon 2020, Marie SkłodowskaCurie IF (project 659668 EVOLPREG), Finnish Cultural Foundation, Jane and Aatos Erkko Foundation, Päivikki and Sakari Sohlberg Foundation, Eemil Aaltonen Foundation, and Sigrid Juselius Foundation, as well as Turku Graduate School (UTUGS), Biocenter Finland, and ELIXIR Finland. Work in the Wagner lab is supported by National Cancer Institute Center Grant (U54-CA209992) and the John Templeton Foundation Grant (\#61329).

\section{Author contribution statement}

K T R conceived and designed the study, conducted the cell cultures and RNA work, analyzed the data, and wrote the manuscript. $\mathrm{T} \mathrm{H}$ participated in the laboratory work and analysis. M M participated in the data analysis and writing of the manuscript. X $M$ participated in the writing of the manuscript. A P contributed in the sampling and participated in the writing. $L \mathrm{~L} E$ provided resources for the work and participated in the writing. M P and G P W participated in the design of the work, writing of the manuscript, and provided resources for the work.

\section{Acknowledgements}

The authors are grateful to Roger Babbitt and Jordan Pober lab (Yale University) for the help with hypoxia cell culture. The authors thank Erica Nyman and Sinikka Collanus from the University of Turku Histology core facility and Satu Orasniemi from the Institute of Biomedicine for their contribution in immunohistochemistry.

\section{References}

Anders S, Pyl PT \& Huber W 2015 HTSeq - a Python framework to work with high-throughput sequencing data. Bioinformatics 31 166-169. (https://doi.org/10.1093/bioinformatics/btu638)

Balamurugan K \& Sterneck E 2013 The many faces of C/EBPS and their relevance for inflammation and cancer. International Journal of Biological Sciences 9 917-933. (https://doi.org/10.7150/ijbs.7224) 
Batie M, Frost J, Frost M, Wilson JW, Schofield P \& Rocha S 2019 Hypoxia induces rapid changes to histone methylation and reprograms chromatin. Science 363 1222-1226. (https://doi.org/10.1126/science.aau5870)

Beste MT, Pfaffle-Doyle N, Prentice EA, Morris SN, Lauffenburger DA, Isaacson KB \& Griffith LG 2014 Molecular network analysis of endometriosis reveals a role for c-Jun-regulated macrophage activation. Science Translational Medicine 6 222ra16. (https://doi.org/10.1126/ scitranslmed.3007988)

Bishop DW 1956 Oxygen concentration in the rabbit genital tract. In Proceedings of the Third International Congress of Animal Reproduction, Physiology, pp 53-55. Ed DW Bishop. London: Brown, Knight \& Truscott.

Bishop T \& Ratcliffe PJ 2014 Signaling hypoxia by hypoxia-inducible factor protein hydroxylases: a historical overview and future perspectives. Hypoxia 2 197-213. (https://doi.org/10.2147/HP.S47598)

Bourdel N, Matsuzaki S, Bazin JE, Pouly JL, Mage G \& Canis M 2007 Peritoneal tissue-oxygen tension during a carbon dioxide pneumoperitoneum in a mouse laparoscopic model with controlled respiratory support. Human Reproduction 22 1149-1155. (https://doi. org/10.1093/humrep/del482)

Chakraborty AA, Laukka T, Myllykoski M, Ringel AE, Booker MA, Tolstorukov MY, Meng YJ, Meier SR, Jennings RB, Creech AL et al. 2019 Histone demethylase KDM6A directly senses oxygen to control chromatin and cell fate. Science 363 1217-1222. (https://doi. org/10.1126/science.aaw1026)

Chang RL, Lin JW, Hsieh DJ-Y, Yeh YL, Shen CY, Day CH, Ho TJ, Viswanadha VP, Kuo WW \& Huang CY 2015 Long-term hypoxia exposure enhanced IGFBP-3 protein synthesis and secretion resulting in cell apoptosis in H9c2 myocardial cells. Growth Factors 33 275-281. (https://doi.org/10.3109/08977194.2015.1077824)

Ding H \& Wu T 2018 Insulin-like growth factor binding proteins in autoimmune diseases. Frontiers in Endocrinology 9 499. (https://doi. org/10.3389/fendo.2018.00499)

Erkenbrack EM, Maziarz JD, Griffith OW, Liang C, Chavan AR, Nnamani MC \& Wagner GP 2018 The mammalian decidual cell evolved from a cellular stress response. PLoS Biology 16 e2005594. (https://doi. org/10.1371/journal.pbio.2005594)

Evans J \& Salamonsen LA 2014 Decidualized human endometrial stromal cells are sensors of hormone withdrawal in the menstrual inflammatory cascade. Biology of Reproduction 90 14. (https://doi.org/10.1095/ biolreprod.113.108175)

Florin L, Hummerich L, Dittrich BT, Kokocinski F, Wrobel G, Gack S, Schorpp-Kistner M, Werner S, Hahn M, Lichter P et al. 2004 Identification of novel AP-1 target genes in fibroblasts regulated during cutaneous wound healing. Oncogene 23 7005-7017. (https://doi. org/10.1038/sj.onc.1207938)

Gellersen B \& Brosens JJ 2014 Cyclic decidualization of the human endometrium in reproductive health and failure. Endocrine Reviews 35 851-905. (https://doi.org/10.1210/er.2014-1045)

Hahne M, Schumann P, Mursell M, Strehl C, Hoff P, Buttgereit F \& Gaber T 2018 Unraveling the role of hypoxia-inducible factor (HIF)-1 $\alpha$ and HIF-2 $\alpha$ in the adaption process of human microvascular endothelial cells (HMEC-1) to hypoxia: redundant HIF-dependent regulation of macrophage migration inhibitory factor. Microvascular Research 116 34-44. (https://doi.org/10.1016/j.mvr.2017.09.004)

Hastings JM, Jackson KS, Mavrogianis PA \& Fazleabas AT 2006 The estrogen early response gene FOS is altered in a baboon model of endometriosis. Biology of Reproduction 75 176-182. (https://doi. org/10.1095/biolreprod.106.052852)

Heinosalo T, Gabriel M, Kallio L, Adhikari P, Huhtinen K, Laajala TD, Kaikkonen E, Mehmood A, Suvitie P, Kujari H et al. 2018 Secreted frizzled-related protein 2 (SFRP2) expression promotes lesion proliferation via canonical WNT signaling and indicates lesion borders in extraovarian endometriosis. Human Reproduction 33 817-831. (https://doi.org/10.1093/humrep/dey026)

Hussein M, Chai DC, Kyama CM, Mwenda JM, Palmer SS, Gotteland JP \& D'Hooghe TM 2016 C-Jun NH2-terminal kinase inhibitor bentamapimod reduces induced endometriosis in baboons: an assessor-blind placebocontrolled randomized study. Fertility and Sterility 105 815.e5-824.e5. (https://doi.org/10.1016/j.fertnstert.2015.11.022)

Jha P \& Das H 2017 KLF2 in regulation of NF-kB-mediated immune cell function and inflammation. International Journal of Molecular Sciences 18 2383. (https://doi.org/10.3390/ijms18112383)
Kajihara T, Brosens JJ \& Ishihara O 2013 The role of FOXO1 in the decidual transformation of the endometrium and early pregnancy. Medical Molecular Morphology 46 61-68. (https://doi.org/10.1007/s00795-013-0018-z)

Kommagani R, Szwarc MM, Kovanci E, Gibbons WE, Putluri N, Maity S, Creighton CJ, Sreekumar A, DeMayo FJ, Lydon JP et al. 2013 Acceleration of the glycolytic flux by steroid receptor coactivator-2 is essential for endometrial decidualization. PLoS Genetics 9 e1003900. (https://doi.org/10.1371/journal.pgen.1003900)

Liu H, Zhang Z, Xiong W, Zhang L, Xiong Y, Li N, He H, Du Y \& Liu Y 2017 Hypoxia-inducible factor- $1 \alpha$ promotes endometrial stromal cells migration and invasion by upregulating autophagy in endometriosis. Reproduction 153 809-820. (https://doi.org/10.1530/REP-16-0643)

Lu Z, Zhang W, Jiang S, Zou J \& Li Y 2014 Effect of oxygen tensions on the proliferation and angiogenesis of endometriosis heterograft in severe combined immunodeficiency mice. Fertility and Sterility 101 568-576. (https://doi.org/10.1016/j.fertnstert.2013.10.039)

Manabe I, Shindo T \& Nagai R 2002 Gene expression in fibroblasts and fibrosis: involvement in cardiac hypertrophy. Circulation Research $\mathbf{9 1}$ 1103-1113. (https://doi.org/10.1161/01.res.0000046452.67724.b8)

Maybin JA \& Critchley HOD 2015 Menstrual physiology: implications for endometrial pathology and beyond. Human Reproduction Update 21 748-761. (https://doi.org/10.1093/humupd/dmv038)

Maybin JA, Murray AA, Saunders PTK, Hirani N, Carmeliet P \& Critchley HOD 2018 Hypoxia and hypoxia inducible factor- $1 \alpha$ are required for normal endometrial repair during menstruation. Nature Communications 9 295. (https://doi.org/10.1038/s41467-017-02375-6)

Mazur EC, Vasquez YM, Li X, Kommagani R, Jiang L, Chen R, Lanz RB, Kovanci E, Gibbons WE \& DeMayo FJ 2015 Progesterone receptor transcriptome and cistrome in decidualized human endometrial stromal cells. Endocrinology 156 2239-2253. (https://doi.org/10.1210/en.20141566)

Muter J, Lucas ES, Chan YW, Brighton PJ, Moore JD, Lacey L, Quenby S, Lam EW-F \& Brosens JJ 2015 The clock protein period 2 synchronizes mitotic expansion and decidual transformation of human endometrial stromal cells. FASEB Journal 29 1603-1614. (https://doi.org/10.1096/ fj.14-267195)

Muter J, Alam MT, Vrljicak P, Barros FSV, Ruane PT, Ewington LJ, Aplin JD, Westwood M \& Brosens JJ 2018 The glycosyltransferase EOGT regulates adropin expression in decidualizing human endometrium. Endocrinology 159 994-1004. (https://doi.org/10.1210/en.2017-03064)

Nancy P, Siewiera J, Rizzuto G, Tagliani E, Osokine I, Manandhar P, Dolgalev I, Clementi C, Tsirigos A \& Erlebacher A 2018 H3K27me3 dynamics dictate evolving uterine states in pregnancy and parturition. Journal of Clinical Investigation 128 233-247. (https://doi.org/10.1172/ JC195937)

Natsuizaka M, Naganuma S, Kagawa S, Ohashi S, Ahmadi A, Subramanian H, Chang S, Nakagawa KJ, Ji X, Liebhaber SA et al. 2012 Hypoxia induces IGFBP3 in esophageal squamous cancer cells through HIF-1 $\alpha$-mediated mRNA transcription and continuous protein synthesis. FASEB Journal 26 2620-2630. (https://doi.org/10.1096/fj.11-198598)

Owusu-Akyaw A, Krishnamoorthy K, Goldsmith LT \& Morelli SS 2019 The role of mesenchymal-epithelial transition in endometrial function. Human Reproduction Update 25 114-133. (https://doi.org/10.1093/ humupd/dmy035)

Pavličev M, Wagner GP, Chavan AR, Owens K, Maziarz J, Dunn-Fletcher C, Kallapur SG, Muglia L \& Jones H 2017 Single-cell transcriptomics of the human placenta: inferring the cell communication network of the maternal-fetal interface. Genome Research 27 349-361. (https://doi. org/10.1101/gr.207597.116)

Piñero J, Bravo À, Queralt-Rosinach N, Gutiérrez-Sacristán A, Deu-Pons J, Centeno E, García-García J, Sanz F \& Furlong LI 2017 DisGeNET: a comprehensive platform integrating information on human diseaseassociated genes and variants. Nucleic Acids Research 45 D833-D839. (https://doi.org/10.1093/nar/gkw943)

Pohnke Y, Kempf R \& Gellersen B 1999 CCAAT/enhancer-binding proteins are mediators in the protein kinase A-dependent activation of the decidual prolactin promoter. Journal of Biological Chemistry 274 24808-24818. (https://doi.org/10.1074/jbc.274.35.24808)

Pringle KG, Kind KL, Sferruzzi-Perri AN, Thompson JG \& Roberts CT 2010 Beyond oxygen: complex regulation and activity of hypoxia inducible factors in pregnancy. Human Reproduction Update 16 415-431. (https:// doi.org/10.1093/humupd/dmp046) 
Rekker K, Saare M, Eriste E, Tasa T, Kukuškina V, Roost AM, Anderson K, Samuel K, Karro H, Salumets A et al. 2017 High-throughput mRNA sequencing of stromal cells from endometriomas and endometrium. Reproduction 154 93-100. (https://doi.org/10.1530/REP-17-0092)

Robinson MD, McCarthy DJ \& Smyth GK 2010 edgeR: a Bioconductor package for differential expression analysis of digital gene expression data. Bioinformatics 26 139-140. (https://doi.org/10.1093/ bioinformatics/btp616)

Russell S, Gatenby RA \& Gillies RJ Pseudohypoxia: Life at the Edge. In: Ecology and Evolution of Cancer. pp 57-68 Academic Press. 2017. (http s://doi.org/10.1016/b978-0-12-804310-3.00004-1).

Rytkönen KT, Erkenbrack EM, Poutanen M, Elo LL, Pavlicev M \& Wagner GP 2019 Decidualization of human endometrial stromal fibroblasts is a multiphasic process involving distinct transcriptional programs. Reproductive Sciences 26 323-336. (https://doi. org/10.1177/1933719118802056)

Salker MS, Nautiyal J, Steel JH, Webster Z, Šućurović S, Nicou M, Singh Y, Lucas ES, Murakami K, Chan YW et al. 2012 Disordered IL-33/ST2 activation in decidualizing stromal cells prolongs uterine receptivity in women with recurrent pregnancy loss. PLOS ONE 7 e52252. (https://doi. org/10.1371/journal.pone.0052252)

Sato F, Bhawal UK, Yoshimura T \& Muragaki Y 2016 DEC1 and DEC2 crosstalk between circadian rhythm and tumor progression. Journal of Cancer 7 153-159. (https://doi.org/10.7150/jca.13748)

Smythies JA, Sun M, Masson N, Salama R, Simpson PD, Murray E, Neumann V, Cockman ME, Choudhry H, Ratcliffe PJ et al. 2019 Inherent DNA-binding specificities of the HIF- $1 \alpha$ and HIF- $2 \alpha$ transcription factors in chromatin. EMBO Reports 20 e46401. (https://doi.org/10.15252/ embr.201846401)

Tal R 2012 The role of hypoxia and hypoxia-inducible factor-1alpha in preeclampsia pathogenesis. Biology of Reproduction 87 134. (https:// doi.org/10.1095/biolreprod.112.102723)

Tamaresis JS, Irwin JC, Goldfien GA, Rabban JT, Burney RO, Nezhat C, DePaolo LV. \& Giudice LC 2014 Molecular classification of endometriosis and disease stage using high-dimensional genomic data. Endocrinology 155 4986-4999. (https://doi.org/10.1210/en.2014-1490)

Tamura I, Shirafuta Y, Jozaki K, Kajimura T, Shinagawa M, Maekawa R, Taketani T, Asada H, Sato S, Tamura H et al. 2017 Novel function of a transcription factor WT1 in regulating decidualization in human endometrial stromal cells and its molecular mechanism. Endocrinology 158 3696-3707. (https://doi.org/10.1210/en.2017-00478)

Trapnell C, Pachter L \& Salzberg SL 2009 TopHat: discovering splice junctions with RNA-Seq. Bioinformatics 25 1105-1111. (https://doi. org/10.1093/bioinformatics/btp120)

Vento-Tormo R, Efremova M, Botting RA, Turco MY, Vento-Tormo M, Meyer KB, Park JE, Stephenson E, Polański K, Goncalves A et al. 2018 Single-cell reconstruction of the early maternal-fetal interface in humans. Nature 563 347-353. (https://doi.org/10.1038/s41586018-0698-6)

Vinketova K, Mourdjeva M \& Oreshkova T 2016 Human decidual stromal cells as a component of the implantation niche and a modulator of maternal immunity. Journal of Pregnancy 2016 8689436. (https://doi. org/10.1155/2016/8689436)
Wade MA, Jones D, Wilson L, Stockley J, Coffey K, Robson CN \& Gaughan L 2015 The histone demethylase enzyme KDM3A is a key estrogen receptor regulator in breast cancer. Nucleic Acids Research $\mathbf{4 3}$ 196-207. (https://doi.org/10.1093/nar/gku1298)

Wagner GP, Kin K \& Lynch VJ 2013 A model based criterion for gene expression calls using RNA-seq data. Theory in Biosciences 132 159-164. (https://doi.org/10.1007/s12064-013-0178-3)

Wang W, Taylor RN, Bagchi IC \& Bagchi MK 2012 Regulation of human endometrial stromal proliferation and differentiation by C/EBP $\beta$ involves cyclin E-cdk2 and STAT3. Molecular Endocrinology 26 2016-2030. (https://doi.org/10.1210/me.2012-1169)

Wu MH, Lu CW, Chang FM \& Tsai SJ 2012 Estrogen receptor expression affected by hypoxia inducible factor- $1 \alpha$ in stromal cells from patients with endometriosis. Taiwanese Journal of Obstetrics and Gynecology $\mathbf{5 1}$ 50-54. (https://doi.org/10.1016/j.tjog.2012.01.010)

Wu SP, Li R \& DeMayo FJ 2018 Progesterone receptor regulation of uterine adaptation for pregnancy. Trends in Endocrinology and Metabolism 29 481-491. (https://doi.org/10.1016/j.tem.2018.04.001)

Wu MH, Hsiao KY \& Tsai SJ 2019 Hypoxia: the force of endometriosis. Journal of Obstetrics and Gynaecology Research 45 532-541. (https:// doi.org/10.1111/jog.13900)

Xue W, Liu Y, Zhao J, Cai L, Li X \& Feng W 2012 Activation of HIF-1 by metallothionein contributes to cardiac protection in the diabetic heart. American Journal of Physiology: Heart and Circulatory Physiology 302 H2528-H2535. (https://doi.org/10.1152/ajpheart.00850.2011)

Yang S, Fang Z, Suzuki T, Sasano H, Zhou J, Gurates B, Tamura M, Ferrer K \& Bulun S 2002 Regulation of aromatase P450 expression in endometriotic and endometrial stromal cells by CCAAT/enhancer binding proteins (C/EBPs): decreased C/EBP $\beta$ in endometriosis is associated with overexpression of aromatase. Journal of Clinical Endocrinology and Metabolism 87 2336-2345. (https://doi.org/10.1210/jcem.87.5.8486)

Yu J, Berga SL, Johnston-MacAnanny EB, Sidell N, Bagchi IC, Bagchi MK \& Taylor RN 2016 Endometrial stromal decidualization responds reversibly to hormone stimulation and withdrawal. Endocrinology 157 2432-2446. (https://doi.org/10.1210/en.2015-1942)

Zhang L, Xiong W, Li N, Liu H, He H, Du Y, Zhang Z \& Liu Y 2017 Estrogen stabilizes hypoxia-inducible factor $1 \alpha$ through $G$ protein-coupled estrogen receptor 1 in eutopic endometrium of endometriosis. Fertility and Sterility 107 439-447. (https://doi.org/10.1016/j.fertnstert.2016.11.008)

Zuo RJ, Gu XW, Qi QR, Wang TS, Zhao XY, Liu JL \& Yang ZM 2015 Warburg-like glycolysis and lactate shuttle in mouse decidua during early pregnancy. Journal of Biological Chemistry 290 21280-21291. (https://doi.org/10.1074/jbc.M115.656629)

Received 20 December 2019

First decision 4 February 2020

Revised manuscript received 27 March 2020

Accepted 9 April 2020 